\title{
Perspectivas del financiamiento corporativo y el mercado de valores del Perú
}

\section{Perspectives on corporate finance and the peruvian stock market}

Dr. Indalecio Enrique Horna Zegarra es Doctor en Ciencias Contables. Decano de la Facultad de Ciencias Económicas, Contables Y Administrativas de la Universidad Nacional de Cajamarca (ihorna@unc.edu.pe) (https://orcid.org/0000-0002-8375-243X)

\begin{abstract}
Resumen
El grado de desarrollo de los mercados financieros son un indicativo del crecimiento económico de los países. Una alternativa para el financiamiento de las empresas es la colocación de bonos corporativos a través del mercado de valores, así, este estudio pretende revisar el comportamiento en el mercado de valores peruano relativo a las emisiones de bonos corporativos comparativamente durante los periodos 2005-2010 y 2015-2019, como reflejo de la situación del mercado actual peruano. Es una investigación de tipo documental y descriptiva, recopilando datos relativos a las colocaciones de bonos corporativos (montos, plazos de vencimiento y tasas de interés), mediante la revisión de notas de prensa de la Superintendencia del Mercado de Valores (SMV) y los respectivos informes acumulados, de las diferentes compañías clasificadoras de riesgo aprobadas por SMV, para cada una de las empresas en estudio. Se observó en promedio un aumento en las emisiones, así como la disminución de las tasas de interés y ambas tendencias, fueron estadísticamente significativas. Por otra parte, dos de las empresas revisadas, emitieron bonos verdes tanto en el mercado nacional como en el internacional. Estos, son indicadores favorables para la economía del Perú, los cuales validan que viene en crecimiento y más aún, predispuestas al cambio global en donde se busca más que el financiamiento empresarial, un impacto positivo en el ámbito social y en el medio ambiente, con la emisión de bonos verdes.
\end{abstract}

\begin{abstract}
The degree of development of financial markets is an indicator of countries' economic growth. An alternative financing for companies is the placement of corporate bonds through the stock market, so this study aims to comparatively review the behavior of the Peruvian stock market in relation to corporate bond issues during the periods 2005-2010 and 2015-2019, as a reflection of the current situation of the Peruvian market. It is a documentary and descriptive study that collects data related to corporate bond placements (amounts, maturity terms and interest rates), through the review of press releases from the Superintendence of the Securities Market (SMV) and the respective cumulative reports of the different risk classifiers approved by the SMV, for each of the companies under study. On average, an increase in emissions was observed, as well as a decrease in interest rates, and both trends were statistically significant. On the other hand, two of the companies examined issued green bonds in both the domestic and international markets. These are favorable indicators for Peru's economy, validating that it is growing and even more so, predisposed to global change in which more than business financing is sought, a positive impact in the social and environmental spheres, through the issuance of green bonds.
\end{abstract}

\section{Palabras clave I keywords}

Mercado de valores, bonos corporativos, bonos verdes, mercado de bonos.

Stock market, corporate bonds, green bonds, bond market.

Cómo citar: Horna Zegarra, I. E. (2020). Perspectivas del financiamiento corporativo y el mercado de valores del Perú. Retos Revista de Ciencias de la Administración y Economía, 10(19), 135152. https://doi.org/10.17163/ret.n19.2020.08 


\section{Introducción}

Variados estudios en economía, relacionan el crecimiento de los mercados financieros con el desarrollo de la economía, dando importancia al beneficio que le genera a un país, el desarrollo y crecimiento de los mercados de capitales. Así, para alcanzar mejores tasas de crecimiento económico es condición necesaria el desarrollo del mercado financiero en el país (Moreno, Vásquez, Hernández \& Larios, 2015; Greenbaun, Thakor \& Boot, 2019).

El mercado de valores es parte del mercado financiero, en el que confluyen diversos agentes económicos como empresas estatales, empresas corporativas, individuos y otros, quienes requieren y entregan fondos prestables. Este mercado tiene como función la de centralizar las negociaciones de valores financieros, llamados también instrumentos financieros.

Las empresas que participan en el mercado para captar recursos son los emisores y aquellas que disponen de capital para financiar, se les llama inversores. Los valores negociables, son las acciones, bonos, instrumentos de corto plazo, etc., y dependiendo del instrumento, este otorga derechos a los inversores, en la participación de las ganancias o dividendos de las empresas, derecho al voto en las juntas de accionistas, o a recibir pagos de intereses (MEF, 2016a).

En el mercado de valores se ofrecen varias alternativas de inversión según las necesidades tengan los emisores o inversores, en cuanto a criterios como el rendimiento, la liquidez y el riesgo. Así el dinero percibido por los emisores es utilizado principalmente para financiar proyectos de inversión o de reestructuración de los pasivos de la empresa (Stowell, 2018).

El mercado de valores, ofrece entonces alternativas de financiamiento, cuando a través de su sistema se otorgan los recursos del ahorro, creándose el canal de asignación de la inversión; reduciendo el costo de intermediación financiera y constituye el comienzo del cambio financiero. Por tanto, cumplen la función de facilitar el proceso de inversión al proporcionar un mercado donde de forma eficiente y con menores costos, se efectúan las transacciones. De tal modo que los inversionistas de forma segura pueden vender y comprar valores si así lo requieren y realizar transacciones continuas, garantizando el precio de los mismos (Stowell, 2018). Ahora bien, muchos inversionistas con alta experiencia en su indagación, retrospectivamente observan como en tiempos pasados se podía obtener ingresos razonables de certificados de depósito relativamente seguros, y fondos del mercado monetario, hoy día se han sentido alentados por lo que parece ser el comienzo de un aumento más rápido en las tasas de interés afectando la estabilidad de los precios en las respectivas inversiones, es por ello que el aumento de las tasas causa una pérdida de atracción por las acciones ya sobrevaluadas mucho antes de que las inversiones de pensiones en los certificados de depósito y los fondos del mercado monetario se convirtieran en inversiones de capital rentable, tanto para los inversores existentes como las generaciones anteriores.

Si una empresa es juzgada favorablemente por los inversionistas, se estima que el valor bursátil se hace más atractivo, se fortalece financieramente la entidad, estos adquieren volúmenes más altos lo que facilita nuevos financiamientos y más 
crecimiento (Jacobsen \& Venkataraman, 2018). Además, se estabiliza la operación del mercado de valores, al generar mercados continuos que provocan cambios de precio más frecuentes, pero más pequeños. En mercados de menor movimiento, los cambios de precio son menos frecuentes, pero más violentos; y esto facilitará la asimilación de las nuevas emisiones de valores y su exitoso lanzamiento (Greenbaun et al., 2019).

A nivel mundial, una alternativa muy utilizada para el financiamiento de los sectores empresariales, son las colocaciones primarias de bonos corporativos, antes de recurrir a un crédito bancario (Rodríguez, 2010; Jacobsen \& Venkataraman, 2018). La colocación o emisión de bonos en el mercado satisface las necesidades financieras de mediano y largo plazo y el capital adquirido se utiliza para financiar las nuevas propuestas de inversión como la adquisición de nuevos activos o la reestructuración de deudas a largo plazo (Rodríguez, 2010; Stowell, 2018; Greenbaun et al., 2019).

La Ley del Mercado de Valores del Perú en el artículo 86 del Decreto Legislativo $\mathrm{N}^{\circ} 861$, define a los bonos como la oferta pública de valores representativos de deuda a plazo mayores a un año, y la Ley de Títulos Valores los incluye dentro de los denominados valores mobiliarios. Donde la oferta primaria pública (OPP), implica que va dirigida al público en general y por primera vez y los emisores, demandantes de fondos, ofrecen valores mobiliarios (acciones, bonos, certificados de depósito negociables, instrumentos de corto plazo, etc.) a los agentes superavitarios que tienen excedente de fondos, los inversionistas.

Para que una empresa ingrese en la OPP debe cumplir con los requisitos exigidos por la normativa que regula el mercado de valores, siendo uno de ellos la inscripción de los valores materia de la oferta en el Registro Público del Mercado de Valores (RPMV), esto, previo a la colocación de dichos valores entre los inversionistas (Acosta, 2017).

El RPMV es un registro administrado por la Superintendencia del Mercado de Valores (SMV), que es el supervisor y regulador del mercado de valores público en el Perú. La inscripción en el RPMV implica poner a disposición del sistema integrado de revelación permanente, toda la información de la empresa al mercado en relación a los valores que se están negociando, lo que favorece la adjudicación del precio de los valores en el mercado y adicionalmente ejerce el efecto de reducir los costos asociados a la revelación de la información (Acosta, 2017).

En general, los empresarios se quejan del acceso limitado que se les presenta en el acceso a mercado primario de capitales (Chalamandaris \& Vlachogiannakis, 2018; Nikolova, Wang \& Wu, 2019). En Perú son pocas las empresas que recurren a la emisión de bonos corporativos, esto debido en parte a la cantidad de requisitos a los cuales están sujetas las ofertas públicas, y en segundo lugar las empresas que pueden acceder al financiamiento a través de estos instrumentos de deuda son calificadas en su mayoría como grandes corporaciones, ya que sus necesidades de financiamiento son tan grandes que diluyen los costos fijos de emisión. La legislación debe promover una mayor competencia a las colocaciones primarias de bonos corporativos, para mejorar el mercado primario de valores, con la idea de que éste se realmente ofrezca una alternativa de financiamiento que compita con el sistema bancario (Rodríguez, 2010).

De hecho, la Ley de Promoción del Mercado de Valores (Ley N 30050), así como el Reglamento del Mercado de Inversionistas Institucionales, aprobado por 
Resolución SMV N N $^{\circ}$ 21-2013-SMV/01, contempla una excepción al régimen para emitir valores que se ofertan públicamente a inversionistas institucionales, en el cual se agregan algunas ventajas para los potenciales emisores. Como menos requisitos para la inscripción y formulación de la oferta de valores (acciones, bonos e instrumentos de corto plazo), menores tiempos de duración de los trámites y menos costo, entre otros beneficios. Lo que representa un cambio, el inicio de nuevas tendencias económicas para el Perú (MEF, 2019a).

Durante los años 2007-2009, se manifestó a nivel global una contracción de la economía; que impactó el mercado local de bonos corporativos, disminuyendo las emisiones y los montos, luego gradualmente, de acuerdo al informe del Ministerio de Economía y Finanzas del Perú (2016) en los últimos años el sector privado ha participado y utilizado el mercado de valores, por lo que éste ha venido creciendo (MEF, 2016b).

Lo expuesto, es motivo para presentar una revisión de las colocaciones de bonos corporativos en las ofertas primarias públicas en el mercado de valores peruano de algunas de las empresas peruanas que por tradición utilizan este medio para su financiamiento, como una extensión que permite conocer los cambios que han venido ocurriendo a la economía peruana.

\subsection{Instrumentos de financiamiento}

Los instrumentos negociados en el mercado de valores son conocidos como valores financieros, activos financieros o simplemente valores. La legislación peruana establece diferencias entre títulos valores y valores mobiliarios, siendo el primero la letra de cambio, el cheque, pagaré; mientras que los valores mobiliarios refieren a las acciones y bonos, certificados de depósito negociables, instrumentos de corto plazo, etc. (Art ${ }^{\circ} 1$ Ley de Títulos Valores (LTV) del 19/06/2000; Art `3 Ley del Mercado de Valores (LMV), Decreto Legislativo No 861, del 22/10/1996).

El decreto legislativo $N^{\circ} 861$ en el artículo 3 de la Ley del Mercado de Valores (LMV), vigente desde el 22/10/1996, establece que son valores mobiliarios aquellos emitidos en forma masiva y libremente negociables que confieren a sus titulares derechos crediticios, dominiales o patrimoniales, o los de participación en el capital, el patrimonio o las utilidades del emisor. Y el artículo 86 define a los bonos como la oferta pública de valores representativos de deuda a plazo mayor de un año.

En el mercado de valores se diferencian varios tipos de bonos dependiendo del agente emisor, entre los que provienen del sector público, están: bonos del tesoro, de capitalización del Banco Central de Reserva, de reconocimiento y Brady; y por parte del sector privado: bonos corporativos, de arrendamiento financiero y subordinado (Noriega,1998; Stowell, 2018).

Los bonos son entonces, los instrumentos financieros de renta fija, emitidos a mediano y largo plazo por un agente deficitario que puede ser una empresa, el gobierno o un organismo público (municipalidad), con una determinada tasa de interés (tasa fija, o cero cupones) y fechas ya fijadas para el pago de los intereses (cupones) y reintegro del principal. Representan una ventaja para los emisores porque no necesariamente están amparados con garantías definidas al contrario de los créditos 
bancarios en los cuales se pueden gravar más frecuentemente los activos fijos como la garantía (Rodríguez, 2010; Pilbeam, 2018; Stowell, 2018).

La emisión de bonos cubre necesidades financieras de mediano y largo plazo y el capital devengado se utilizará para financiar parte de sus activos fijos, proyectos de inversión o la reestructuración de los compromisos fijados a largo plazo. Este aspecto es precisamente lo que diferencia de otros instrumentos de corto plazo, los cuales están dirigidos para cubrir necesidades financieras de corto plazo (Sambola, 2012).

Entre los bonos emitidos por el sector privado, están los bonos de arrendamiento financiero, que son emitidos por empresas autorizadas a realizar operaciones de arrendamiento financiero (leasing) a un plazo no menor a tres años pudiendo ser colocados bajo el par y ofrecer un rendimiento fijo o variable. Si es necesario, no hay impedimento para incorporar como garantía de la emisión cualquier activo de la entidad emisora, de terceros o una carta fianza otorgada por una entidad del sistema financiero (MEF, 2012).

Los bonos subordinados, son emitidos por bancos y empresas financieras a plazos mayores de cinco años, estos no tienen la opción de garantías, ni se pueden cancelar antes de su vencimiento ni el rescate por sorteo. Si la empresa emisora entra en liquidación, son redimidos luego de cumplir con las demás obligaciones a cargo del emisor. Los bonos subordinados, sólo se anticipan a las acciones comunes y preferenciales en el orden de prelación (Sambola, 2012; MEF, 2012).

Y los bonos corporativos, objeto de este estudio, que son los emitidos por las empresas para captar fondos que le permitan financiar sus operaciones y proyectos de inversión. Son emitidos a su valor nominal el cual será pagado al tenedor en la fecha de vencimiento. El monto devenga un interés que se cancela en cuotas periódicas (cupones) o íntegramente en la fecha de vencimiento, que será mayor a un año (Rodríguez, 2010; Pilbeam, 2018).

\subsection{Mercado de fondos prestables}

Invertir, representa destinar recursos hacia activos que aumentarán la capacidad de producción de una corporación. Lo que esta invierte depende de la tasa de interés, que es el costo del préstamo. Por supuesto la empresa, solo aceptará proyectos cuyas ganancias sean mayores al costo de financiamiento. De aquí que, a mayor tasa de interés, menor será la demanda de los préstamos de una empresa determinada. Así la interacción entre las funciones de oferta y demanda determinarán la tasa de interés de equilibrio (Arrarte, 2018).

Desde el punto de vista financiero, la inversión es una operación en la cual, se aporta inicial o totalmente el nivel de activos requeridos en un determinado proceso de producción u otorgamiento de servicios. Su esencia descansa en el deseo de sacrificar consumo presente, con la finalidad de acceder a un mayor consumo en el futuro.

Que un agente inversor tenga la posibilidad de financiar a otros o que como emisor requiera recibir financiamiento, depende en parte de la relación entre la inversión y el ahorro en un momento determinado. Cuando en una empresa hay escasez de recursos financieros (agentes deficitarios), el agente tenderá a financiarse a través de los diversos instrumentos que ofrecen los mercados financieros (Arrarte, 2018). 
Los mercados de fondos prestables (o mercados de capitales) son los canales mediante los cuales los agentes deficitarios, obtienen el recurso financiero para adquirir sus activos fijos. Estos recursos provienen del ahorro de los agentes superavitarios. Una característica del mercado de fondos prestables es que se efectúan toda clase de inversiones o transacciones relativas a capitales o activos financieros (compra venta de acciones, bonos, entre otros) así como la colocación de préstamos en el largo plazo (García, 2014). En este mercado, es donde se ajusta el precio del capital, para hacer que la cantidad ofrecida sea igual a la cantidad demandada, estableciéndose la tasa de interés. Así, la tasa de interés es el precio de uso de fondos prestados durante un período específico (Gómez González, Huertas, Cristiano \& Chavarro, 2016; Pilbeam, 2018).

Por tanto, en el mercado de capitales se negocian los instrumentos financieros de relativamente largo plazo, facilitando la unión que se establece entre las disposiciones para el ahorro versus la inversión que tienen las empresas involucradas. Así, el valor del activo financiero se adjudica por el precio actual de los flujos de caja esperados (es el dinero líquido que se espera recibir durante el periodo prefijado de dicha inversión financiera) (García, 2014).

De acuerdo con la teoría de la ley de la oferta y demanda, entonces los compradores de bonos están dispuestos a comprar más bonos a un precio menor. Cuanto menor sea el precio del bono, más alto será su rendimiento al vencimiento. Cuanto más alto sea el rendimiento al vencimiento, más agentes superavitarios estarán dispuestos a prestar dinero (Chalamandaris \& Vlachogiannakis, 2018). Y en contraposición, los prestatarios de bonos ofrecerán más bonos a un precio más alto. Precios de bonos más altos significan rendimientos al vencimiento menores. Los oferentes de bonos están dispuestos a ofrecer más bonos (tomar más fondos) a tasas de interés más bajas que a tasas de interés más altas. (Pilbeam, 2018)

La oferta y la demanda de bonos determinan como se cotiza el mismo. Así, en la medida que la tasa de interés del mercado disminuye, el precio del bono será mayor y viceversa (Arrarte, 2018; Pilbeam, 2018).

\section{Materiales y método}

El objetivo de este estudio es realizar una revisión retrospectiva de las colocaciones de bonos corporativos de algunas empresas que participan en el mercado de valores peruano durante los periodos 2005-2010 y 2015-2019, con la finalidad de valorar la situación financiera que presentan dichas empresas en ambos periodos en correspondencia con la dinámica de cambios que ha ocurrido en la economía peruana.

Se trata de una investigación de tipo documental y descriptiva, ya que se ubicaron los datos consultando diferentes tipos de documentación, así la información correspondiente al periodo 2005-2010 corresponde a los datos del trabajo de investigación doctoral del autor del presente estudio, titulada "Colocaciones primarias de bonos corporativos en el mercado de valores peruano". Y la obtención de los datos del periodo 2015-2019, se realizó mediante la revisión de noticias de prensa emitidas por la Superintendencia del Mercado de Valores y los respectivos informes emitidos por las diferentes compañías clasificadoras de riesgo como: Class \& Asociados 
Clasificadora de Riesgos, PCR Pacific Credit Rating, Apoyo y Asociados fitch ratings, y Equilibrium Clasificadora de Riesgo, para cada una de las empresas en los informes acumulados año 2019. Ubicando la información concerniente a los montos de las colocaciones de bonos corporativos anuales, el plazo de vencimiento y las tasas de interés.

El muestreo fue no probabilístico dirigido, ya que se escogieron 10 empresas a discreción de la condición de encontrar la información de la empresa en relación a la colocación de bonos corporativos en el mercado de valores, el plazo de vencimiento y las tasas de interés promedio al valor nominal anual, en ambos periodos, ya sea publicada en las redes sociales a través de noticias de prensa o en los documentos de dominio público, que se encuentran en los portales de la Superintendencia del Mercado de Valores (SMV) y en la web como los informes de las clasificadoras de riesgo. Los datos de los años 2011-2014, no se incluyeron en el estudio, debido a que en ese periodo algunas empresas no emitieron bonos o la información no estaba disponible.

A los datos recopilados se les aplicó estadística descriptiva, tabulando en gráficas de barras los montos y las tasas. Y adicionalmente, se les aplicó estadística inferencial, empleando la prueba no paramétrica de Pearson para el análisis de la tendencia de los montos colocados, mientras que se empleó el índice de Spearman para valorar la tendencia con respecto a las tasas de interés, con el fin de corresponder dichas tendencias a la situación que actualmente presenta la economía del Perú.

\section{Resultados}

Durante el periodo 2005 al 2010, un total de 70 empresas realizaron ofertas públicas primarias en el mercado de valores peruano, de las cuales 46 empresas fueron las que recurrieron al financiamiento a través de bonos corporativos, representando el $66 \%$ del total colocado en soles, seguido de un $18 \%$ y $8 \%$ en certificados de depósito negociables e instrumentos de corto plazo, respectivamente. Del total de colocaciones en soles y en dólares, el 55\% se efectuó en nuevos soles y la diferencia en dólares (45\%) (Horna, 2012).

En el gráfico 1, se muestran los montos totales acumulados por cada una de las empresas en los periodos 2005-2010 y 2015-2019, observándose en términos generales un aumento del total de emisiones en soles en la mayoría de las empresas, liderado por la compañía Alicorp con un aumento en sus emisiones en US\$322 millones, equivalentes a 1094 millones de soles. Y como excepción la compañía ENEL Distribución Perú, con una disminución de US\$ 81 millones, equivalentes a 274.31 millones de soles en el acumulado de sus colocaciones en referido periodo. 


\section{Gráfico 1. Montos en US\$ totales colocados por empresas a través de bonos corporativos en el mercado de valores peruano en los periodos 2005-2010 y 2015-2019}

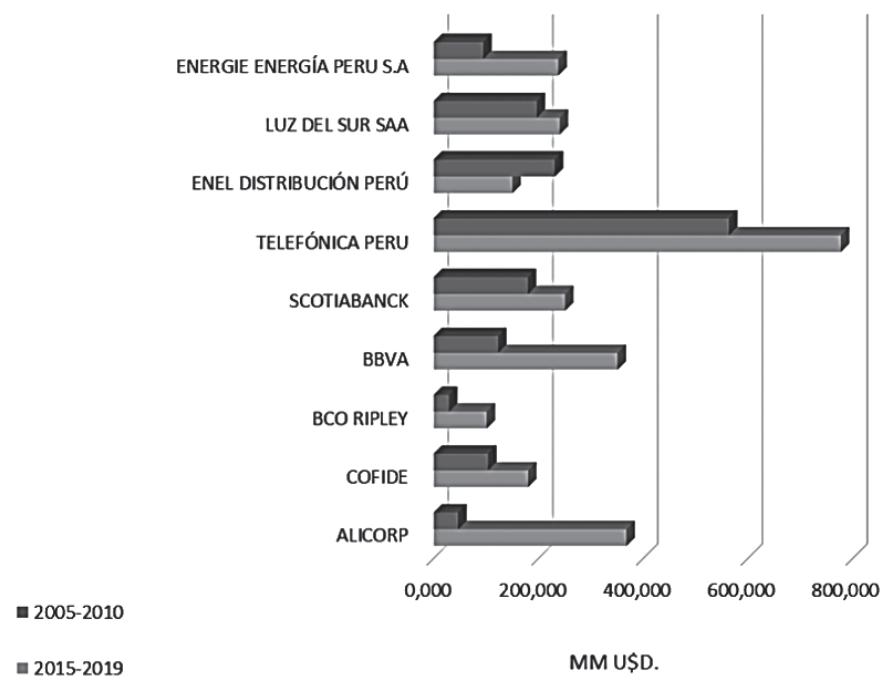

Fuente: Elaboración propia, con datos obtenidos de los informes de las clasificadoras de riesgos para cada una de las empresas citadas: Cariñaupa y Barba (2019); Córdova (2019); Garro (2018); Garro (2019); Izquierdo y Gallo (2018a, 2018b); Tarazona y Regis (2019); Tejada y Barba (2019); Tejada y Regis (2019); Tejada y Vallejo (2019).

Al aplicar a los datos el estadístico inferencial de Pearson, para correlacionar la tendencia entre ambos periodos, se obtuvo un índice $\mathrm{R}=0,80$, indicando que las colocaciones de bonos en los dos periodos presentan una correlación lineal muy fuerte y positiva. Es decir, que la tendencia de las empresas es hacia el incremento de los montos de las colocaciones de bonos corporativos en el mercado de valores.

Con respecto a la tasa de interés promedio a la que se fijaron las cuotas de vencimiento de los diferentes plazos, en el gráfico 2 se observa de manera general que la misma disminuye al comparar los periodos estudiados. Las empresas que no se ajustaron a esta tendencia fueron ALICORP, Luz del Sur y Telefónica Perú, con diferencias de 0.1;0.4 y 0.9 puntos respectivamente. Destacándose el caso de Telefónica Perú, cuyo aumento en la tasa promedio fue la mayor.

Así mismo al realizar el análisis de correlación de la tendencia en los dos periodos estudiados, se observó que esta no fue lineal, ajustando mejor los datos al modelo de Spearman (no lineal) arrojando en este caso un índice $R=-0,74$, negativo y con un valor alto que indica correlación muy fuerte, es decir, que las tasas de interés fijado a las emisiones de bonos corporativos presentan una tendencia a disminuir, con un índice de correlación negativo y muy fuerte. 
Por otra parte, en este estudio además de las empresas privadas escogidas, se incluyeron con finalidad comparativa, a dos empresas bancarias estadales públicas, como la Corporación Andina de Fomento (CAF) ahora Banco de Desarrollo de América Latina y la Corporación Financiera de Desarrollo S.A. (COFIDE) (ver tabla 1), las cuales en sus actividades económicas representan a las políticas de estado, dando las pautas de alguna forma a la economía en el mercado.

La CAF, no se incluyó en las gráficas anteriores, pues para el periodo 20052010 de colocar un monto total aproximado de US\$116 millones (393 millones de soles) en bonos corporativos, presentó un giro considerable en el reciente periodo, en donde todas sus colocaciones fueron en 12 tipos de moneda extranjera en mercado internacional, que alcanzaron un monto total de US\$ 4900 millones, de los cuales US \$ 130 millones correspondieron a tres colocaciones de Bonos Verdes en el 2018. Y, por otra parte, COFIDE, quien sí ha venido participando en la oferta publica primaria del mercado de valores peruano, colocando bonos corporativos, los cuales han marcado tendencia al aumento del valor de los montos, pasando aproximadamente en su equivalente en dólares de US\$ 104099000 para el periodo 2005-2010 a US\$180 091 000 en el 2015-2019, presentando en el primer trimestre del 2019 un giro al colocar US $\$ 100$ millones en Bonos Verdes, a un plazo de tres años con una tasa de $5.13 \%$.

Gráfico 2. Tasa de interés promedio a la que se fijaron las emisiones de bonos corporativos total acumulado en el mercado de valores peruano en los periodos 2005-2010 y 2015-2019

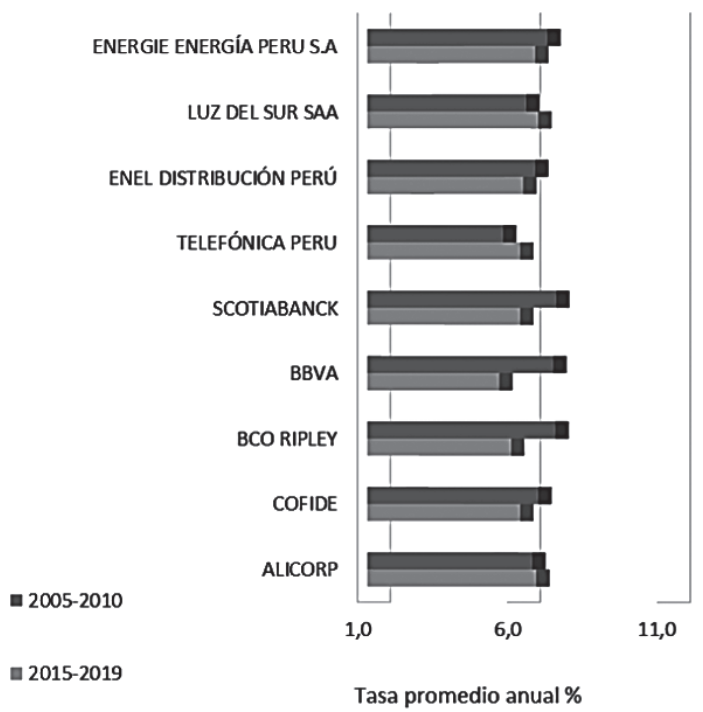

Fuente: Elaboración propia, con datos obtenidos de los informes de las clasificadoras de riesgos para cada una de las empresas citadas: Cariñaupa y Barba (2019); Córdova (2019); Garro (2018); Garro (2019); Izquierdo y Gallo (2018a, 2018b); Tarazona y Regis (2019); Tejada y Barba (2019); Tejada y Regis (2019); Tejada y Vallejo (2019). 


\section{Tabla 1. Colocación de bonos corporativos de las empresas CAF y COFIDE durante el periodo 2015-2019}

\begin{tabular}{|c|c|c|c|c|c|}
\hline Empresa & Tipo de bono & $\begin{array}{l}\text { Año de } \\
\text { emisión }\end{array}$ & $\begin{array}{l}\text { Monto } \\
\text { acumulado } \\
\text { MM US\$ }\end{array}$ & $\begin{array}{l}\text { Plazo } \\
\text { promedio } \\
\text { (años) }\end{array}$ & $\begin{array}{c}\text { Tasa de } \\
\text { interés \% } \\
\text { promedio }\end{array}$ \\
\hline \multirow[t]{2}{*}{$\begin{array}{l}\text { Corporación } \\
\text { Andina de Fo- } \\
\text { mento (CAF) }\end{array}$} & $\begin{array}{l}\text { Corporativo en } \\
\text { mercado internacio- } \\
\text { nal, Colocación en } \\
\text { dólares } \\
\end{array}$ & $\begin{array}{l}\text { Acumulado } \\
\text { 2015-2019 }\end{array}$ & 21020 & 3 & 2.12 \\
\hline & Bonos Verdes & 2018 & 130 & 6 & 3.99 \\
\hline \multirow{2}{*}{$\begin{array}{l}\text { Corporación } \\
\text { Financiera } \\
\text { de Desarrollo } \\
\text { (COFIDE) }\end{array}$} & $\begin{array}{l}\text { Corporativos merca- } \\
\text { do nacional }\end{array}$ & $\begin{array}{l}\text { Acumulado } \\
2015-2018\end{array}$ & 180,691 & 9 & 6.28 \\
\hline & Bonos verdes & 2019 & 29,41 & 3 & 5.13 \\
\hline
\end{tabular}

Fuente: Elaboración propia con datos de S\&P Global, (2017); Krmelj, Regis y Tejada, 2019.

\section{Conclusiones y discusión}

Para los países, el mercado primario de valores representa un importante rol en su economía, pues facilita la reducción de los costos de transacción, al obtener un financiamiento con menores tasas de interés. Durante el periodo 2005-2010, la mayoría de las empresas que conformaban al sector empresarial peruano presentaban volúmenes de activos reducidos, por lo que el requerimiento de financiamiento no es lo suficientemente grande como para poder entrar al mercado primario de valores con un costo que sea menor al que representan otras alternativas de financiamiento, representando una limitante estructural. De hecho, de acuerdo con SMV para el año 1999, de un total de 1828 empresas en el Perú, solo un 2\% presentaban activos mayores de US $\$ 200$ millones.

En el periodo 2005-2010 hubo limitadas emisiones de empresas corporativas, las cuales, al captar todo el flujo de capital, contribuían en cierta medida a reducir la diversificación del mercado de capitales y a la concentración de colocaciones de los activos financieros. Esto, dado al bajo número de empresas dispuestas a participar de la OPP, independientemente de que requieran financiamiento y a que esta sea considerada una de las mejores alternativas por los bajos costos de emisión, pero en este tipo de financiamiento las empresas están sometidas a un marco jurídico que regula las operaciones.

Como expresado anteriormente, las empresas para participar en la OPP de valores deben inscribirse en el RPMV y solicitar su participación ante SMV, anexando la documentación e información que se indica en la Ley del Mercado de Valores, Decreto Legislativo $\mathrm{N}^{\mathrm{o}} 861$ y sus normas modificatorias. Uno de los documentos que se debe presentar es un proyecto de prospecto informativo. El prospecto informativo es un documento legalmente vinculante que contiene toda la información financiera necesaria, para que el inversionista pueda tomar una decisión de inversión informada. De la inscripción al RPMV las empresas quedan en la obligación de poner a 
disposición del registro de sistema integrado de revelación permanente toda la información al mercado respecto de los valores. Esto para un importante sector empresarial peruano se considera una limitante; por lo que, del total de empresas que hay en Perú, muy pocas acceden a esta alternativa a la hora de buscar financiamiento, la mayoría, han demostrado ser renuentes a proporcionar su información al mercado; es decir, no desean hacer pública la información referente a la marcha de sus empresas. Debido tal vez, a una débil o poca cultura de revelación de información financiera, que de igual manera incide en la baja apreciación a dicha instrucción, desde el punto de vista tributario y fiscal.

En este sentido, sin embargo, se contraponen los resultados del estudio realizado por Nikolova, Wang y Wu (2019), para empresas de los Estados Unidos de América, en su estudió observó que estas empresas se quejan del acceso limitado a los mercados primarios, basado en la poca información que de las empresas emisoras se dispone.

En el caso peruano, otra limitante para la participación de las empresas, a las OPP de bonos corporativos, son los costos de acceso; debido a que el mínimo valor de las emisiones debe ser mayor de US $\$ 10$ millones para que una empresa valorada con nivel de riesgo AAA contemple rentable emitir deuda, de lo contrario, sus gastos fijos no los podría cubrir. Este monto mínimo de emisión es un obstáculo que le impide a las empresas de menor capital participar en el mercado, pues requieren de montos menores de financiamiento.

Así, a partir del 2010 la SMV, ha promovido un conjunto de iniciativas conducentes a fortalecer el rol del mercado de valores en el financiamiento del desarrollo económico del Perú, buscando desde el punto de vista de la oferta, promover el ingreso de nuevos emisores, flexibilizando la regulación, nuevos mecanismos, manteniendo visibilidad de la información y conocimiento de los riesgos, garantizando la protección de los inversionistas y permitiendo la entrada de nuevos inversionistas. La SMV registró el ingreso aproximado de 31 nuevos emisores al mercado de capitales durante el periodo 2010 al 2015 y en este sentido, en el 2012 se creó el Mercado Alternativo de Valores (MAV) dirigido a empresas no corporativas (Roca, 2016), todo con la finalidad de sentar las bases para el crecimiento del mercado.

Durante el período 2005-2010, del total de colocaciones en el mercado de capitales, el 55\% se efectuó en soles frente a un 45\% realizado en dólares. Esta preferencia de financiamiento en soles aminora el riesgo cambiario en la estructura de los balances. Dado por el fortalecimiento del dólar, la caída de precio de los commodities y la volatilidad del mercado cambiario, afectando negativamente las inversiones y colocaciones en moneda extranjera.

Este comportamiento, se siguió repitiendo a lo largo de los años, la SMV, reportó en el 2011, el incremento de las colocaciones en soles en un 62\%, mientras que el 38\% restante correspondió a las colocaciones realizadas en dólares (MEF, 2016b). Asimismo, en el 2015, solo se realizó una emisión de bono corporativo en dólares por el monto de US\$97.3 millones por la Administradora Jockey Plaza Shopping Center S.A. representando, el 28.5\% del total emitido en todos los instrumentos de deuda para la fecha (Bonos Corporativos, Subordinados, Arrendamiento Financiero, etc.) (Vallejo \& Barbieri, 2016). 
Esta conducta se repite en el primer semestre del 2019, cuando las colocaciones de bonos en dólares sólo representaron el 7\%; mientras que, las colocaciones en soles representaron el 93\%. Lo que sigue indicando una fuerte confianza en el mercado interno. (MEF 2019b)

En relación a los datos de este estudio, se obtuvo una correlación fuerte y positiva con $\mathrm{R}=8,0$, en cuanto a las colocaciones de bonos corporativos en soles en el mercado de valores entre los periodos 2005-2010 y 2015-2019, indicando que la tendencia al aumento del número de emisiones de bonos por parte del sector empresarial es estadísticamente significativa, lo que demuestra efectivamente, la confianza que este sector demuestra frente al país y el fortalecimiento de su economía.

Así, todas las empresas evaluadas aumentaron el monto total colocado a excepción de ENEL Distribución Perú, esto es debido a que lo que se está expresando es la colocación en soles, más sin embargo actualmente esta empresa cuenta con un respaldo a través de préstamos "intercompany", hasta por un importe máximo de US $\$ 200$ millones con un plazo que finaliza en diciembre del 2020. Para las empresas que conforman el grupo en Perú, este monto les facilita un eficiente manejo de los fondos disponibles. Este grupo es una de las principales multinacionales del mercado eléctrico y de gas, que opera en 35 países de los cinco continentes. En Perú tiene la concesión de la distribución y comercialización de energía eléctrica en la zona norte de Lima Metropolitana, en la Provincia Constitucional del Callao y las provincias de Huaura, Huaral, Barranca y Oyón; atendiendo a 52 distritos de manera exclusiva y compartiendo cinco distritos adicionales con la empresa distribuidora de la zona sur de Lima, beneficiando a más de la mitad de los pobladores de Lima Metropolitana (Garro, 2019a).

En cuanto a las variaciones de las tasas de interés en ambos periodos, se obtuvo una correlación fuerte y negativa con $\mathrm{R}=-0.7$, lo que indica que la disminución de las tasas de interés entre ambos periodos, 2005-2010 y 2015-2019, es estadísticamente significativo. Y esto se explica por el hecho de que, al haber mayores emisiones por mayores montos, e incluso un aumento en el número de emisores, más confianza se genera en el mercado por los inversores, lo que conlleva a la baja de las tasas de interés (Robles, Sutton \& Vtyurina, 2017).

De todas las empresas revisadas, en solo tres se observó un aumento de la tasa de interés, siendo más significativo el caso de la empresa Telefónica de Perú, con una diferencia de 1.9 más que el promedio de tasas obtenido para el periodo 20052010 (Garro, 2018). Esto fue debido que sus colocaciones de bonos corporativos en el periodo 2015-2019 rondaron en plazos de vencimiento entre 5 y 8 años, mientras que sus colocaciones en el periodo anterior fueron a plazos más largos entre 10 y 20 años y además por montos menores, variables que influyen en el mercado para la determinación de las tasas de interés (Robles et al., 2017).

En ese sentido, uno de los problemas más difíciles que atraviesa cualquier economía en estos últimos meses es el elevado costo del crédito. La crisis recesiva que atraviesa cualquier país podría ser menos dura si bajara la tasa de interés en forma más rápida, como con cualquier otro precio en las diversas economías de mercado, los tipos de interés son determinadas por las fuerzas de la oferta y de la demanda, en este caso, de la oferta y la demanda de crédito. Si la oferta de crédito (S) de los 
prestamistas aumenta con relación la demanda (D) de los prestatarios, el precio (tipo de interés) tenderá para bajar mientras que los prestamistas compiten para encontrar el uso para sus fondos (Rivas, 2012).

Por otra parte, Galicial, Cárdenas y Rivas (2017) reportan de acuerdo a un estudio en relación a los países que conforman la bolsa de valores del mercado integral latinoamericano (MILA), los cuales son Colombia, Chile, Perú y México. Señalan que Perú siendo el país con una de las economías más bajas en relación al resto de países que conforman MILA, estando incluso 5.6 veces por debajo de México, cuenta con $1.9 \%$ más de empresas que cotizan en el mercado de valores. En el estudio, Perú se ubica en segundo lugar, como país con más cantidad de empresas bursátiles, y al compararse con México demuestra que presenta una cultura que se inicia hacia la apertura, pues comparativamente se observan más empresas dispuestas a mostrar su información, por otra parte, comparativamente tiene más empresas que contribuyen al sector primario, conducta que resulta coherente para ser un país considerado en vías de desarrollo.

Robles et al. (2017) realizaron también un estudio comparativo sobre el mercado de capitales en los países de América Latina, señalando que es Perú seguido de Brasil, los países en los cuales principalmente las negociaciones se basan en la emisión de bonos corporativos principalmente en sus mercados nacionales. Y en el caso de Perú, expresamente dominadas por instituciones financieras y unas pocas empresas energéticas.

La globalización financiera ha abierto oportunidades de financiación favorables para las empresas con sede en estos países, ahora denominados emergentes, aunque no muchas empresas tengan el tamaño y la capacidad de gestión necesarios para emitir bonos internacionales, no obstante, la creciente emisión de bonos, como fuente de fondos para nuevas inversiones está funcionando como un complemento de otros mecanismos, como el de la inversión extranjera directa (López-Herrera, Santillán-Salgado, \& Cabello, 2019).

Es innegable que la economía peruana viene creciendo, luchando contra la contracción por la crisis mundial, y la misma situación de política interna, más, sin embargo, ha sabido salir adelante manteniendo niveles de inflación bajos y estables. Sin embargo, aún falta mucho por hacer, así Galicial et al. (2017) encuentra que son Chile y Perú los países que tienen las tasas de impuestos mayores en 2 y 3 puntos por encima de otros, en lo que respecta a los costos por emisión de instrumentos en el mercado y es este régimen de exención fiscal, el que ha desincentivado a los empresarios a participar del mercado, lo que genera un efecto negativo en el ámbito empresarial de cada país.

Cuando las grandes empresas participan en el mercado de valores nacional, para financiarse, colocando grandes cantidades en soles, reflejan el dinamismo en la inversión privada, así como demuestran la confianza en el plan país, por lo que, así como es una forma de canjear su deuda en dólares y reperfilar los plazos de sus amortizaciones, para evitar el riesgo cambiario, igualmente representan un fuerte apoyo al mercado interno.

Las empresas buscan financiamiento para poder llevar a cabo sus nuevos proyectos de expansión, crecimiento de sus activos o inversión en adelantos tecnológicos, 
en el caso de entidades financieras los recursos se lo ofrecen a sus clientes en calidad de préstamos, pero recientemente a nivel mundial, surgen nuevas tendencias, originadas en la necesidad de proteger el planeta, buscando alternativas que contribuyan a preservar la naturaleza, en lucha contra el calentamiento global. Surge entonces los bonos verdes, los cuales serán instrumentos de deuda con plazos mayores a un año, diferenciándose del resto en que los fondos captados son utilizados exclusivamente al financiamiento de proyectos nuevos o ya en curso, los cuales tengan un impacto ambiental positivo, es decir, que el bono se emite con el fin determinado de invertir en proyectos concernientes con el medio ambiente y el cambio climático (The World Bank, 2015).

En términos generales, los bonos verdes son valores de renta fija emitidos para la captación de capital, por entidades, para financiar sus proyectos respetuosos con el medio ambiente, como la energía renovable, la gestión sostenible del agua, la prevención de la contaminación, adaptación al cambio climático y así sucesivamente (Tang \& Zhang, 2018).

El Banco Europeo de Inversiones y el Banco Mundial fueron los dos primeros emisores de bonos verdes, en 2007, para financiar infraestructura ambiental. Sin embargo, este mercado internacionalmente despega en 2013, cuando la Corporación Financiera Internacional (CFI) vendió un bono verde de US\$ 1 mil millones, abriéndose este mercado de emisores en una variedad de sectores y con calificaciones crediticias variables. Se ha iniciado con emisiones de alto rendimiento, pero se espera que aumenten a medida que más corporaciones participen (Flaherty, Gevorkyan, Radpour \& Semmler, 2017).

En el 2014, los bancos inversionistas Bank of America Merrill Lynch, Citi, JPMorgan, BNP Paribas, y HSBC, establecieron los principios de los bonos verdes (GBP; de sus siglas en inglés), entre ellos: la transparencia y divulgación, junto a los cuatro componentes: uso de los ingresos, proceso de la evaluación y selección de proyectos, la gestión de los ingresos y la presentación de informes. Siendo ampliamente aceptadas por el mercado internacional. Independientemente de la generalidad de la GBP, proporciona un criterio de elegibilidad y una taxonomía verde detallada por sector que los terceros pueden adoptar para evaluar la calificación de un bono verde (Tang \& Zhang, 2018).

Entre las empresas que se revisaron en este estudio, se incluyeron dos, las cuales emitieron bonos verdes. Las empresas CAF y COFIDE, siendo las primeras empresas financieras estadales en emitir bonos verdes, con la finalidad de emplear los recursos en proyectos que promuevan la transformación productiva sostenible y la infraestructura verde, como la producción de energía renovable (eólicas, fotovoltaica y pequeñas centrales hidroeléctricas); tecnologías de la información y comunicación; manejo forestal sostenible; transporte limpio; acuicultura y agricultura sostenible y saneamiento como tratamiento de aguas residuales.

En la XXI Conferencia Internacional sobre Cambio Climático (Conferencia de las Naciones Unidas sobre Cambio Climático 2015 celebrada en París-Francia), Perú fue calificado como "país particularmente vulnerable", en dicha conferencia se logró firmar un acuerdo donde se establecen medidas de reducción de las emisiones de gases de efecto invernadero por medio de la mitigación, adaptación y resiliencia de los ecosistemas como consecuencia del calentamiento global. Y ya, en el 2014, 
en Perú, la empresa Rímac Seguros y Reaseguro, invierte adquiriendo los primeros bonos verdes con CFI. Así que las emisiones de bonos verdes realizadas por CAF y COFIDE, son consideradas un avance que continúa impulsando este tipo de iniciativas, esperando que el mercado de bonos verdes vaya ganando mayor promoción y consolidación (Quispe, 2018).

La tendencia global va dirigida a promover y dar importancia a todos los proyectos que tengan impacto positivo en el medio ambiente, Así la Bolsa de Valores de Lima-Perú, la Embajada Británica en el Perú, la Bolsa Mexicana de Valores y Climate Partners participaron en la elaboración de la Guía de bonos verdes para el Perú, la cual se aprobó el 30 de abril del 2018 que busca orientar a las empresas e instituciones para que puedan emitir este tipo de instrumento (Quispe, 2018; Monasterolo \& Raberto, 2018; Flammer, 2018).

A nivel global, si bien las emisiones de bonos verdes han sido inicialmente por financieras, bancos de desarrollo, así como los inversionistas has sido institucionales como administradoras de fondo de pensiones, oficinas de normalización previsional o aseguradoras, lo que se espera es la toma de conciencia y la consolidación de este mercado, en el cual no solo se emitan valores mobiliarios sino de patrimonios, de titulación y otros, junto a la inclusión de corporaciones o empresas como inversionistas (Flammer, 2018).

De acuerdo al estudio de Tang y Zhang (2018) al revisar las emisiones de bonos verdes junto con las iniciativas de inversión denominadas iniciativa de cambio climático (CBI; de sus siglas en inglés) en los cinco continentes. Observaron que las cotizaciones bursátiles aumentan significativamente en torno al anuncio de la emisión de bonos verdes y la reacción de los mercados bursátiles es más fuerte por primera vez que para los emisores repetidos y más fuerte para los emisores corporativos que para los emisores de las instituciones financieras. La emisión de bonos verdes puede ayudar a ampliar la base de inversores, ya que la emisión de bonos verdes atrae más exposición mediática y puede ser utilizada por los inversores de impacto para satisfacer sus mandatos de inversión (Tang \& Zhang, 2018).

Se trata de un mercado todavía relativamente incipiente y sustancialmente más pequeño que los de los bonos convencionales. Y su crecimiento promueve la sostenibilidad al acelerar la capitalización del mercado de bonos verdes; pudiéndose ver los resultados a través de mejoras macroeconómicas (e institucionales), en la búsqueda de la sostenibilidad a través de la financiación de bonos verdes; este crecimiento es capaz de salvar la brecha entre los factores económicos y la sostenibilidad y de reducir los costos de las empresas en la búsqueda de inversiones en sostenibilidad mediante la promoción de emisión de los bonos verdes como alternativas de financiación a un menor costo (Tolliver, Keeley \& Managi, 2019).

Perú, se encuentra en buen camino, haciendo esfuerzos, como el de introducir cambios en los reglamentos (Ley N/³0050, aprobada en resolución SMV Nº21-2013SMV/01: reducción de trámites y de costos en la emisión de valores en el mercado) para hacerlos más abiertos y participativos y así fomentar el ingreso de más empresas al mercado de valores, y el de incrementar las alternativas de financiamiento las cuales dirijan los esfuerzos hacia la protección del medio ambiente, en donde se financien proyectos que mejoren la huella ambiental de la empresa y contribuyan a 
la creación de valor a largo plazo, ayudando a inversionistas que sean sensibles al medio ambiente.

\section{Referencias}

Acosta, M. (2017). El prospecto informativo en las ofertas públicas primarias de valores mobiliarios. Superintendencia del Mercado de Valores (Nota de prensa), Gestión,17 de diciembre 2017. Recuperado de https://bit.ly/2HdOjSD (2019-08-10).

Arrarte, R. (2018). Tasas de interés real neutrales y las normas internacionales de información financiera. Quipukamayoc, 25(49), 9-25. https://doi.org/10.15381/ quipu.v25i49.14276.

Cariñaupa, L., \& Barba, H. (2019). Banco Ripley Perú S.A. Informe de clasificación, Equilibrium clasificadora de riesgos S.A., 11 de junio de 2019, Recuperado de: https://bit.ly/2zftkuk [Fecha de consulta: 13 de agosto de 2019].

Chalamandaris, G., \& Vlachogiannakis, N. (2018). Adverse-Selection Considerations in the Market-Making of Corporate Bonds. SSRN Electronic Journal. DOI:10.2139/ssrn.3209196

Córdova, P. (2019). Fundamento de clasificación de riesgo Luz del Sur S.A.A. Class \& Asociados S. A. Clasificadora de Riesgos, 25 de febrero de 2019 pp. 13 Recuperado de: https://bit. ly/2KLm146 [Fecha de consulta: 09 de agosto de 2019].

Flaherty, M., Gevorkyan, A., Radpour, S., \& Semmler, W. (2017). Financing climate policies through climate bonds. A three stage model and empirics. Research in International Business and Finance. 42. 468-479. https://doi.org/ 10.1016/j.ribaf.2016.06.001

Flammer, C. (2018). Corporate green bonds. SSRN Electronic Journal, 41, julio. http://dx.doi. org/10.2139/ssrn.3125518

Galicial, S., Cárdenas, M., \& Rivas, L. (2017). Las bolsas de valores de los países afiliados al Mercado Integral Latinoamericano (Colombia, Chile, Perú y México). Memoria del XI Congreso de la Red Internacional de Investigadores en Competitividad, 1819-1833; Guadalajara, México: Red Internacional de Investigadores en Competitividad. Recuperado de: https://bit. ly/2Z6BapC

García, V. (2014). Introducción a las finanzas. México, México: ebook. Grupo editorial Patria S.A. de C.V. Recuperado de: https://bit.ly/2McxwlD

Garro, S. (2018). Informe de clasificación de riesgo, Telefónica Perú S.A.A, Class \& Asociados, clasificadora de riesgos, 15 de marzo del 2018, pp. 23. Recuperado de: https://bit.ly/2TOHqfF [Fecha de consulta: 13 de agosto de 2019].

Garro, S. (2019). Informe de clasificación de riesgo, ENEL Distribución Perú S.A.A. Class \& Asociados, clasificadora de riesgos, 08 de mayo de 2019, pp. 18. Recuperado de: https:// bit.ly/2Mtlztp [Fecha de consulta: 13 de agosto de 2019].

Gómez, J., González E., Huertas, C., Cristiano, D., \& Chavarro, X. (2016). Evaluación de la transmisión de la tasa de interés de referencia a las tasas de interés del sistema financiero colombiano. Ecos de economía, 20(42), 19-45. DOI:10.17230 /ecos.2016.42.2

Greenbaun, S., Thakor, A., \& Boot, A. (2019). Contemporary financial intermediation. London, United Kingdom: Elsevier Academic Press.

Horna, I. (2012). Colocaciones primarias de bonos corporativos en el mercado de valores peruano. (Tesis Doctoral) Universidad Nacional de San Marcos, Perú.

Izquierdo, J., \& Gallo, D. (2018a). Scotiabank Perú S.A.A. Informe trimestral, Apoyo \& Asociados Fitch Ratings, 12 de junio de 2018 pp. 15. Recuperado de: https://bit.ly/2P43MuV [Fecha de consulta: 12 de agosto de 2019].

Izquierdo, J., \& Gallo, D. (2018b). BBVA Continental Informe anual, Apoyo \& Asociados Fitch Ratings, 02 de marzo de 2019 pp. 15. Recuperado de: https://bit.ly/2ZmlcTL [Fecha de consulta: 12 de agosto de 2019].

Jacobsen, S., \& Venkataraman, K. (2018). Does Trade Reporting Improve Market Quality in an Institutional Market? Evidence from 144a Corporate Bonds. SSRN. Recuperado de: http:// 
dx.doi.org/10.2139/ssrn.3171056

Krmelj, L., Regis, H., \& Tejada, M. (2019). Corporación Financiera de Desarrollo S.A. COFIDE. Informe de Clasificación. Equilibrium Clasificadora de Riesgo, 02 de julio de 2019, pp. 11. Recuperado de: https://bit.ly/2Hfazf0 [Fecha de consulta: 13 de agosto de 2019].

López-Herrera, F., Santillán-Salgado, R.J., \& Cabello A. (2019). Latin American Corporate Emerging Markets Bond Indices (CEMBIs): Their recent evolution. Global Finance Journal, 41, 104-112. https://doi.org/10.1016/j.gfj.2019.03.002

MEF (2012). Manual de Instrumentos Financieros. Ministerio de Economía y Finanzas, Dirección General de Endeudamiento y Tesoro Público, 31 de julio de 2012. Recuperado de https://bit.ly/3306ZlJ [Fecha de consulta: 06 de agosto de 2019].

MEF (2016a). Capítulo I. Conceptos Básicos sobre el Mercado de Valores. Ministerio de economía y finanzas. Recuperado de: https://bit.ly/2O0lwU3 [Fecha de consulta: 6 de agosto de 2019]

MEF (2016b). Capítulo II. Situación actual del mercado de valores peruano Ministerio de economía y finanzas. Recuperado de: https://bit.ly/2Z4ISAs [Fecha de consulta: 6 de agosto de 2019].

MEF (2019a). SMV aprobó un trámite de inscripción del Programa de Bonos Corporativos hasta por S/ 500 millones para inversionistas institucionales. Nota de Prensa $N^{\circ} 11$ SMV, 26 de junio de 2019. Recuperado de: https://bit.ly/2ZlNcH7 [Fecha de consulta: 6 de agosto de 2019].

MEF (2019b). Empresas obtienen financiamiento en el mercado de valores por más de US $\$ 1,000$ millones en lo que va de este año. Nota de Prensa No 14 SMV, 05 de agosto de 2019. Recuperado de: https://bit.ly/2ZlNcH7 [Fecha de consulta: 6 de agosto de 2019].

Moreno, E., Vásquez, D., Hernández, S., \& Larios, L. (2015). Interdependencia de los mercados de valores en el mundo. Economía: teoría y práctica, 43, 155-181. Recuperado de https:// goolnk.com/xrWP3l

Monasterolo, I., \& Raberto, M. (2018). The EIRIN Flow-of-funds Behavioral Model of Green Fiscal Policies and Green Sovereign Bonds. Ecological Economics. 144, 228-243. https:// doi.org/10.1016/j.ecolecon.2017.07.029

Nikolova, S., Wang, L., \& Wu, J. (2019). Institutional Allocations in the Primary Market for Corporate Bonds. Journal of Financial Economics. Recuperado de: http://dx.doi.org/10.2139/ ssrn. 3181983

Noriega, F. (1998). La Bolsa de Valores, Instituciones e Instrumentos del Mercado de Valores Peruano. Lima, Perú: Universidad de San Martín de Porres.

Pilbeam, K. (2018). Finance and Financial markets. London, England: Macmillan Publishers Limited.

Quispe, F. (2018). Un reto y una oportunidad para la economía y la infraestructura. El mercado de bonos verdes. Jurídica, Suplemento de análisis legal del peruano, 03 de julio 2018. Recuperado de: https://bit.ly/30mhDOn [Fecha de consulta:10 de agosto de 2019].

Rivas, P. (2012). Algunos efectos de la expansión monetaria en las tasas de interés del mercado de préstamos. Pensamiento crítico, 17(2), 137-153. https://doi.org/10.15381/pc.v17i2.8938

Robles, A., Sutton, B., \& Vtyurina S. (2017). Patrones e impulsores de los bonos corporativos en América Latina. Monetaria, Centro de Estudios Monetarios Latinoamericanos, CEMLA, 39(2), 295-348. Recuperado de: https://bit.ly/2Hg 7POH

Roca, L. (2016). Avances normativos con respecto a la emisión de valores en el Perú. Jornadas sobre Tendencias Internacionales de Regulación y Supervisión Financiera en Iberoamérica. SMV, pp.16 Recuperado de: https://bit.ly/2KIoP21

Rodríguez, V. (2010). Ofertas públicas primarias de bonos corporativos en el Perú. Quipukamayoc, 17(33), 71-95. https://doi.org/10.15381/quipu.v17i33.4554

Sambola, R. (2012). Nuevas tendencias en finanzas corporativas. La financiación de las operaciones corporativas. Revista de contabilidad y dirección, 15(12), 65-96. Recuperado de https://bit.ly/2MuYUwO

S\&P Global (2017). Análisis Corporación Andina de Fomento, América Latina. S\& P. Global Ratings, 25 de julio de 2017. pp. 21. Recuperado de: https://bit.ly/2KUJleM (2019-08-13). 
Stowell, D. (2018). Investment Banks, Hedge Funds, and Private Equity. London, United Kingdom: Academic Press.

Tang, D. Y., \& Zhang, Y. (2018). Do shareholders benefit from green bonds? Journal of Corporate Finance. https://doi.org/10.1016/j.jcorpfin.2018.12.001

Tarazona, J., \& Regis, H. (2019). Alicorp S.A.A.Informe de clasificación, Equilibrium Clasificadora de Riesgo S.A., 28 de mayo de 2019. Recuperado de: https://bit.ly/30xCf6J [Fecha de consulta: 09 de agosto de 2019].

Tejada, M., \& Barba, H. (2019). Scotiabank Perú S.A.A.Informe de clasificación, Equilibrium Clasificadora de Riesgo S.A., 26 de marzo de 2019. Recuperado de: https://bit.ly/2NmkXFG [Fecha de consulta: 12 de agosto de 2019].

Tejada, M., \& Regis, H. (2019). BBVA Banco continental S.A. Informe de clasificación, Equilibrium Clasificadora de Riesgo S.A., 27 de marzo de 2019. Recuperado de: https://bit. ly/2NmkXFG [Fecha de consulta: 12 de agosto de 2019].

Tejada, M., \& Vallejo, R. (2019). ENGIE Energía Perú, Informe de clasificación de riesgo, Equilibrium Clasificadora de Riesgo S.A., 22 de mayo de 2019. Recuperado de: https://bit. ly/2qAxfit [Fecha de consulta: 09 de agosto de 2019].

Tolliver, C., Keeley, A.R., \& Managi, S. (2019). Drivers of green bond market growth: The importance of Nationally Determined Contributions to the Paris Agreement and implications for sustainability. Journal of Cleaner Production. https://doi.org/10.1016/j.jclepro.2019.118643

The World Bank (2015). What are Green Bonds? International Bank for Reconstruction and Development. Recuperado de: https://bit.ly/2YotGOv [Fecha de consulta: 16 de agosto de 2019].

Vallejo, R., \& Barbieri, R. (2016). Comportamiento de los Bonos Corporativos en el Mercado Peruano. Equilibrium Clasificadora de Riesgo S.A., abril. Recuperado de: https://bit.ly/2MuSA8x [Fecha de consulta 6 de agosto de 2019]. 